\title{
Isolation and comparison of mesenchymal stem cell-like cells derived from human gastric cancer tissues and corresponding ovarian metastases
}

\author{
KUNYAN ZHOU ${ }^{1}$, MAN XIA $^{2,3 *}$, BO TANG $^{1}$, DARONG YANG ${ }^{1,4}$, NIANLI LIU ${ }^{4}$, DIHONG TANG ${ }^{2}$, \\ HAILONG XIE ${ }^{5}$, XIAOHONG WANG ${ }^{1,4}$, HAIZHEN ZHU ${ }^{1,4}$, CHEN LIU $^{3}$ and CHAOHUI ZUO ${ }^{1,3^{*}}$ \\ ${ }^{1}$ Department of Gastroduodenal and Pancreatic Surgery, Laboratory of Digestive Oncology,
} Hunan Cancer Hospital and Affiliated Cancer Hospital of Xiangya Medical School; ${ }^{2}$ Department of Gynaecological Oncology, Hunan Cancer Hospital and Affiliated Cancer Hospital of Xiangya Medical School, Central South University, Changsha, Hunan 410013, P.R. China; ${ }^{3}$ Department of Pathology, Immunology and Laboratory Medicine, Shands Cancer Center, University of Florida, Gainesville, FL 32610-0275, USA; ${ }^{4}$ Department of Molecular Medicine, College of Biology,

State Key Laboratory of Chemo/Biosensing and Chemometrics, Hunan University, Changsha, Hunan 410082;

${ }^{5}$ Cancer Research Institute, University of South China, Hengyang, Hunan 421001, P.R. China

Received February 11, 2015; Accepted December 2, 2015

DOI: $10.3892 / \mathrm{mmr} .2015 .4735$

\begin{abstract}
Mesenchymal stem cell (MSC)-like cells have been isolated from various types of tumor. It has previously been reported that MSCs are involved in tumorigenesis and its prognosis. The aim of the present study was to isolate and compare MSC-like cells from human gastric cancer (GC) and its metastatic deposits in ovarian tissue. MSC-like cells were isolated from human gastric cancer (hGC-MSCs) and the corresponding ovarian metastatic tissues (hGCOM-MSCs) from 40 patients. The characteristics of hGC-MSCs and hGCOM-MSCs, including their morphology, surface antigens, specific gene expression and differentiation potential, were
\end{abstract}

Correspondence to: Professor Chaohui Zuo, Department of Gastroduodenal and Pancreatic Surgery, Laboratory of Digestive Oncology, Hunan Cancer Hospital and Affiliated Cancer Hospital of Xiangya Medical School, Central South University, 283 Tongzipo Road, Changsha, Hunan 410013, P.R. China

E-mail: zuochaohui@vip.sina.com

Dr Man Xia, Department of Gynaecological Oncology, Hunan Cancer Hospital and Affiliated Cancer Hospital of Xiangya Medical School, Central South University, 283 Tongzipo Road, Changsha, Hunan 410013, P.R. China

E-mail: xiamansummer@sina.com

*Contributed equally

Abbreviations: MSC, mesenchymal stem cells; GC, gastric cancer; HBM, human bone marrow

Key words: mesenchymal stem cells, gastric cancer, metastatic ovarian cancer, differentiation, microenvironment similar to those of MSCs derived from human bone marrow (hBM-MSCs) but different from GC cells. In conclusion, the present study demonstrated that MSC-like cells could be isolated from GC tissue and its ovarian metastatic tissues. The existence of MSC-like cells in GC tissues and its ovarian metastatic tissues suggests that they may be a potential target for cancer therapy, and provides an experimental foundation for investigating their role in the initiation and progression of ovarian metastasis of GC.

\section{Introduction}

Gastric cancer (GC) is the fourth most frequently occurring cancer worldwide and the second leading cause of cancer-associated mortality $(1,2)$. A 2011 analysis demonstrated that 989,600 new GC cases and 738,000 mortalities were estimated to have occurred in 2008 worldwide (3). The occurrence of GC varies with geographical area, with the highest incidence rate of GC in East Asia, particularly in South Korea, Mongolia, Japan and China (4). At present, no effective treatment is available for this disease and identification of early stage GC is difficult as it is often asymptotic or misdiagnosed. In addition, the prognosis of patients with advanced GC remains poor due to its high metastatic recurrence (5) and the complex molecular mechanisms underlying metastasis are not well characterized (6). Clinical observations reveal that even following radical surgery for early GC, $\sim 50 \%$ of patients suffer from recurrence and metastasis. Local recurrence and metastatic disease are also the main causes of mortality of patients with advanced GC. Therefore, inhibition of GC metastasis is an important therapeutic strategy. The main obstacle in treating metastatic disease is that the tumor cells of the primary and metastatic lesions have biological heterogeneity, which presents differences in antigenic properties, drug sensitivity, and the ability to invade and metastasize (7). The 
prognosis of ovarian metastases, also known as Krukenberg tumors, is known to be poor. Krukenberg tumors occur in 0.3-6.7\% of GC patients who undergo surgery and its incidence is significantly higher in autopsies of GC patients (33-41\%) (8). It has been reported that the incidence of Krukenberg tumors, detected during the follow-up observation period following radical gastric resection or secondary surgery, is 3-4\% and has a median survival time of 12-17 months (9). There are relatively few studies on the incidence, treatment or metastatic mechanisms of Krukenberg tumors.

Mesenchymal stem cells (MSCs), also termed mesenchymal stromal cells, belong to a category of clinically relevant cell types that have the potential to be utilized for cell-based therapies, as complicated culturing or handling techniques are not required to yield clinically useful quantities. Traditionally, MSCs are characterized as having tri-lineage potential, that is they can be induced to differentiate into three mesenchymal lineages: Osteoblasts, adipocytes and chondrocytes, but they may also potentially produce other skeletal tissue cells by culturing MSCs under defined mechanochemical conditions (10). MSCs are characterized by the expression of cell surface markers, including CD73, CD90 and CD105, and the absence of expression of hematopoietic lineage markers (11). There has been heightened interest in the capacity of MSCs to home and migrate into tumors (12). The effects of MSCs within tumors are varied. The theory that MSCs promote tumor growth and metastasis has been supported from studies of angiogenesis, tumor cell survival, the immunosuppressive microenvironment, as well as maintenance of cancer stem cells (CSCs) and construction of their mesenchymal niche (13). Nevertheless, it is important to understand the principles and mechanisms through which MSCs regulate tumor progression since these may give insight into the ways in which MSCs may be used to treat tumors. Tumors are able to disseminate systemically to initiate metastatic niches in distant target organs. These niches, composed of bone marrow-derived MSCs, provide permissive conditions for future metastases (14). Bone marrow MSCs promote stem cell dormancy in lung cancer (15), breast cancer (16) and prostate cancer (17) cells in a metastatic niche.

MSC-like cells have been isolated from endometrial cancer (18), glioma $(19,20)$, bone sarcoma (21) and colorectal cancer (22). However, MSC-like cells have not yet been demonstrated in human ovarian metastases of GC. The present study identified and characterized MSC-like cells from ovarian metastases of GC.

\section{Materials and methods}

Isolation of MSC-like cells from human GC tissues and corresponding ovarian metastases. The present study was approved by the Institutional Review Board of Hunan Cancer Hospital and Affiliated Cancer Hospital of Xiangya Medical School (Changsha, China). All human materials were obtained with informed consent and approved by the Ethics Committee of the Hunan Cancer Hospital and Affiliated Cancer Hospital of Xiangya Medical School. Between January 2002 and December 2008, patients with ovarian metastasis of GC diagnosed pathologically at the Hunan Cancer Hospital were recruited. These human tissues were collected and examined with strict adherence to the protocol approved by this hospital. A total of 40 fresh $\mathrm{GC}$ tissues and corresponding ovarian metastatic tissue of gastric origin were collected from 40 female GC patients, with ages ranging between 43 and 80 years (median, 62.5 years), who had initially undergone resection at the Hunan Provincial Cancer Hospital (Changsha, China). The specimens included: Tumor tissue, adjacent normal tissue (at least $5 \mathrm{~cm}$ beyond the primary tumor margin), distant normal tissue and lymph nodes. The fresh tissue specimens were collected, washed with phosphate-buffered saline (PBS), cut into 1-mm $\mathrm{m}^{3}$-sized pieces and maintained in Dulbecco's modified Eagle's medium with low glucose (Gibco; Thermo Fisher Scientific, Inc., Waltham, MA, USA) containing $10 \%$ fetal bovine serum (FBS), penicillin (100 U/ml; Beijing Chemeebio Pharma-tech Co., Ltd., Beijing, China) and streptomycin (100 $\mu \mathrm{g} / \mathrm{ml}$; Beijing Chemeebio Pharma-tech Co., Ltd.). The tissues were subsequently incubated at $37^{\circ} \mathrm{C}$ in humid air with $5 \% \mathrm{CO}_{2}$. The medium was replaced every 3 days after the initial plating. When adherent fibroblast-like cells appeared after 10 days of culture, the attached cells were trypsinized and passaged (without dilution) into a new flask for further expansion. The cells of passage 4 were used for the experiments described. MSCs isolated from human bone marrow (hBM-MSCs) and the GC cell line BCG-823 from our institute were selected as controls and used for evaluation of the experimental results.

Growth curves. The growth curves of human gastric cancer-MSCs (hGC-MSCs), human gastric cancer ovarian metastatic tissue-MSCs (hGCOM-MSCs) and hBM-MSCs at passage 4 were seeded in 24 -well plates $(8,000$ cells/well), followed by counting the number of cells per well on 14 successive days. The procedure was repeated three times and their growth curves were compared.

Flow cytometric analysis. hGC-MSCs and hGCOM-MSCs $\left(2.0 \times 10^{6}\right.$ cells) were trypsinized, washed twice with PBS and immunostained for $30 \mathrm{~min}$ on ice. A minimum of $5 \times 10^{4}$ cells (in $100 \mu \mathrm{lBS} / 0.5 \% \mathrm{BSA} / 2 \mathrm{mmol} / 1 \mathrm{EDTA}$ ) were incubated with the following mouse anti-human monoclonal antibodies: CD73-FITC (561254), CD90-PE (555596), CD105-APC (561443), CD34-FITC (561819), CD45-APC-H7 (347463) and HLA-DR-FITC (555558; all from BD Biosciences, Franklin Lakes, NJ, USA). Labeled cells were analyzed using a flow cytometer (FACS Calibur; BD Biosciences).

Animal studies. All animal experiments were undertaken in accordance with the National Institutes of Health Guide for the Care and Use of Laboratory Animals (National Institutes of Health, Bethesda, MD, USA), with the approval of the Hunan Provincial Cancer Hospital Review Board and Affiliated Cancer Hospital of Xiangya Medical School and also following Hunan province government Animal Care and Used Committee-approved protocols. A total of eight, 6-week-old female nonobese diabetic (NOD)/severe combined immunodeficiency mice (SCID; Laboratory Animal Center of Shanghai, Academy of Sciences, Shanghai, China) were housed in individually ventilated cages and maintained in a standard animal facility under controlled environmental conditions, 
Table I. Primer sequences for the amplification of target and control cDNAs.

\begin{tabular}{lllc} 
Gene & \multicolumn{1}{c}{ Forward primer } & \multicolumn{1}{c}{ Reverse primer } & $\begin{array}{c}\text { Product } \\
\text { size }(\mathrm{bp})\end{array}$ \\
\hline ALP & TAAGGACATCGCCTACCAGCTC & TCTTCCAGGTGTCAACGAGGT & 170 \\
DMP-1 & GTGAGTGAGTCCAGGGGAGATAA & TTTTGAGTGGGAGAGTGTGTGC & 111 \\
OSC & TGAGAGCCCTCACACTCCTC & ACCTTTGCTGGACTCTGCAC & 98 \\
OPN & CAGTTGTCCCACAGTAGACAC & GTGATGTCCTCGTCTGTAGCATC & 127 \\
Bmi-1 & GCTGCCAATGGCTCAATG & AGGAGACTGCACTGGAGTACTG & 530 \\
ABCG2 & CGGCTTGCAACAACTATGAC & ATCCTGCTTGGAAGGCTCTA & 537 \\
Nanog & ATGCCTCACACGGAGACTG & CTGCGTCACACCATTGCTA & 369 \\
$\alpha$-SMA & CTGACTGAGCGTGGCTATTC & CCACCGATCCAGACAGAGTA & 452 \\
CD44 & TCACAGGTGGAAGAAGAGAC & CATTGCCACTGTTGATCACT & 447 \\
CD73 & CTCGGCTCTTCACCAAGGTT & AATTTGGCCTCTTTGAGGAGT & 226 \\
$\beta$-actin & CGTCTGGACCTGGCTGGCCGGGACC & CTAGAAGCATTTGCGGTGGACGATG & 600
\end{tabular}

ALP, alkaline phophatase; DMP-1, dentin matrix acidic phosphoprotein 1; OSC, osteocalcin; OPN, osteopontin; ABCG2, ATP-binding cassette sub-family G member 2; $\alpha$-SMA, $\alpha$-smooth muscle actin; CD, cluster of differentiation.

at room temperature $26 \pm 2^{\circ} \mathrm{C}$. BCG- 823 , hGC-MSCs or hGCOM-MSCs cells $\left(5 \times 10^{6}\right)$ in $200 \mu$ l PBS were injected subcutaneously into the lower right flank of mice. The incidence of tumor formation was observed over 4 weeks.

Osteogenic differentiation in vitro. hGC-MSCs, hGCOM-MSCs or hBM-MSCs were seeded at 5,000 cells $/ \mathrm{cm}^{2}$ in $35 \mathrm{~mm}$ diameter plates and cultured in DMEM with $10 \%$ FBS, with or without osteogenic supplements $(0.1 \mathrm{nM}$ dexamethasone, $10 \mathrm{mM} \beta$-glycerophosphate, $50 \mathrm{mg} / \mathrm{l}$ ascorbic acid and $4 \mu \mathrm{g} / \mathrm{ml}$ basic fibroblast growth factor; Sigma-Aldrich, St. Louis, MO, USA). The medium was changed three times each week and the cells were induced for 3 weeks. At the end of induction, the cells were subjected to alkaline phosphatase (ALP) staining followed by hematoxylin counterstaining (Zhingshan Golden Bridge, Beijing, China).

Reverse transcription polymerase chain reaction (RT-PCR). Total cellular RNA was isolated from hGC-MSCs and hGCOM-MSCs using TRIzol reagent (Invitrogen; Thermo Fisher Scientific, Inc.), according to the manufacturer's instructions. A total of $1.0 \mu \mathrm{g}$ RNA was processed for cDNA synthesis with Superscript II reverse transcriptase using Oligo-dT orimers (Toyobo Co., Ltd., Osaka, Japan). PCR was performed using $1 \mu \mathrm{g}$ of the cDNA sample with $0.3 \mathrm{U}$ Taq polymerase (CinnaGen Co., Tehran, Iran) and $200 \mu \mathrm{M}$ dNTPs, $10 \mathrm{pM}$ of each primer, reaction buffer, and $\mathrm{MgCl}_{2}$ (Takara Bio Inc., Otsu, Japan). A total of $1 \mu \mathrm{l}$ of this mixture was used as a template for PCR to assess mRNA expression of ALP, dentin matrix acidic phosphoprotein 1 (DMP-1), osteocalcin (OSC) and osteopontin (OPN) using the ABI PRISM 7700 instrument (Applied Biosystems; Thermo Fisher Scientific, Inc., Foster City, CA, USA) with gene-specific primers [Table I, and described previously $(23,24)]$ and the SYBR Green I (Thermo Fisher Scientific, Inc.) protocol. The PCR amplification was performed for 35 cycles, with the cycling conditions as follows: $94^{\circ} \mathrm{C}$ for $30 \mathrm{sec}, 60^{\circ} \mathrm{C}$ (primer) for $30 \mathrm{sec}, 72^{\circ} \mathrm{C}$ for $30 \mathrm{sec}$, with a final extension at $72^{\circ} \mathrm{C}$ for $10 \mathrm{~min}$. PCR products were separated on a $1 \%$ agarose gel, stained with ethidium bromide and visualized under UV light. Relative expression ratios normalized to that of $\beta$-actin (Actb) were calculated.

Statistical analysis. All results are expressed as the mean \pm standard deviation or median (range). The normality of data distribution was assessed by the Kolmogorov-Smirnov test. Data analysis was performed using SPSS version 16.0 (SPSS, Inc., Chicago, IL, USA). Receiver operating characteristic (ROC) curves were generated to determine the areas under the ROC curves and their $95 \%$ confidence intervals were calculated. A univariate test was used to examine the effect of each clinical variable on survival. Student's t-test was applied to determine statistical significance. $\mathrm{P}<0.05$ was considered to indicate a statistically significant difference.

\section{Results}

Morphology of hGC-MSCs and hGCOM-MSCs. The first plastic-adherent cells were detected 1 week after tissue preparation. Primary cell cultures began to form colonies after 10 days of primary culture and reached a confluence of 90-95\% at day 30 (Fig. 1A). Cells from human GC tissues and its ovarian metastatic tissues were seeded at low density on a 6-well plate and allowed to grow until $80 \%$ confluence. Cells were passaged at the same cell density.

Growth curves. The growth curves of hGCOM-MSCs, hGC-MSCs and hBM-MSCs are shown in Fig. 1B. Within 5 days after plating, the number of hGCOM-MSCs increased by almost two times that of hGC-MSCs and hBM-MSCs. In addition, hGCOM-MSCs demonstrated a higher cumulative population doubling level compared with hGC-MSCs and hBM-MSCs.

Surface markers and gene expression in hGC-MSCs and hGCOM-MSCs. Specific cell-surface markers were selected to evaluate whether the hGC-MSCs and hGCOM-MSCs at 


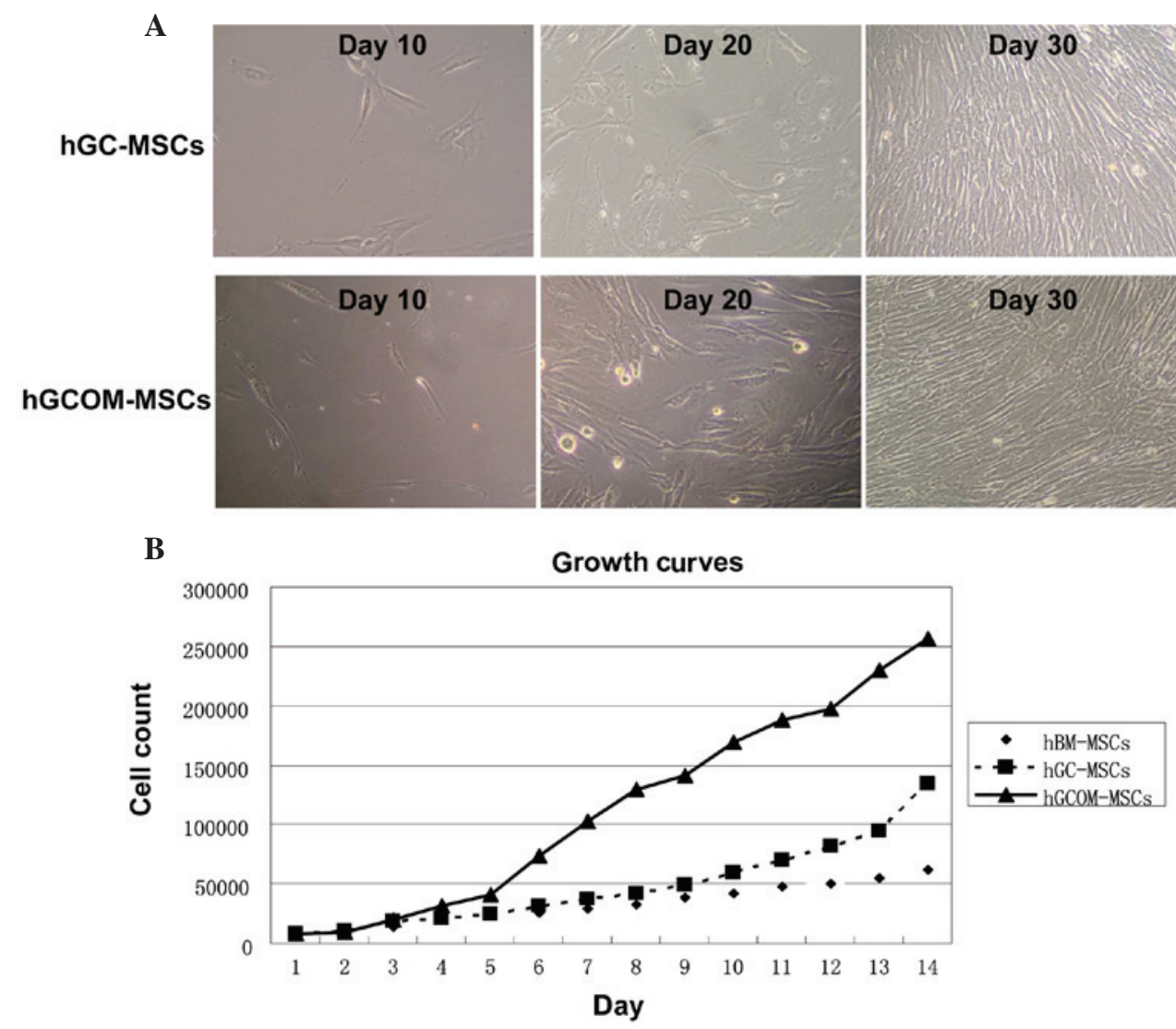

Figure 1. Morphology, growth and the cell cycle of hGC-MSCs, hGCOM-MSCs and normal MSCs. (A) Morphology of hGC-MSCs and hGCOM-MSCs. The isolated MSC-like cells resemble long, spindle-shaped fibroblasts. Cell colony formation of hGC-MSCs and hGCOM-MSCs at passage 0 on the indicated days (magnification, x100). (B) Growth curves of hGC-MSCs and hGCOM-MSCs. hBM-MSCs served as a control. MSCs, mesenchymal stem cells; hGC-MSCs, human gastric cancer-MSCs; hGCOM-MSCs, human gastric cancer ovarian metastatic tissue-MSCs; hBM-MSCs, human bone marrow-MSCs.

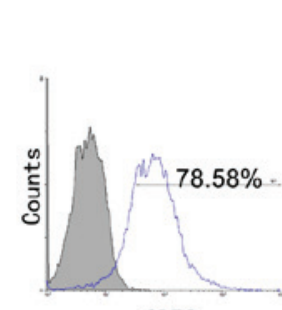

CD73

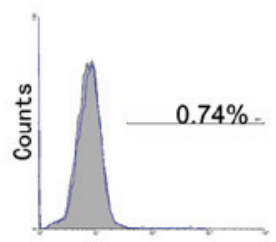

CD34

\section{hGC-MSCs}

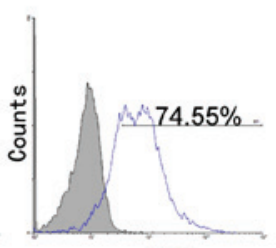

CD90

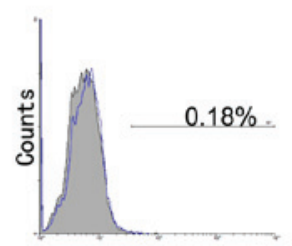

CD45

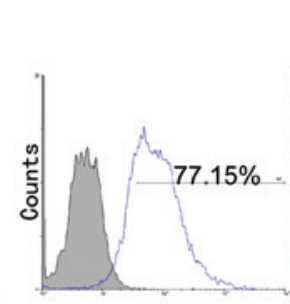

CD105

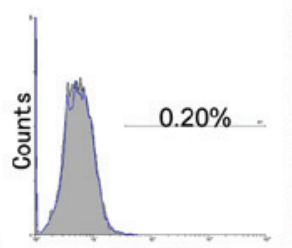

HLA-DR

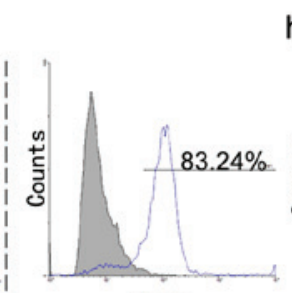

CD73

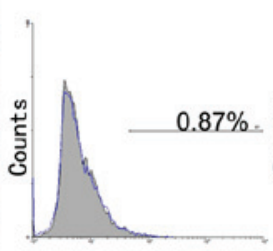

CD34

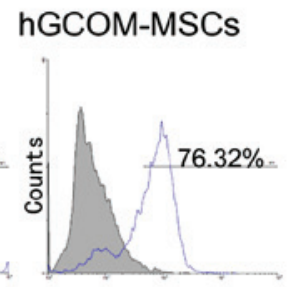

CD90

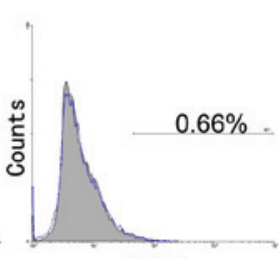

CD45

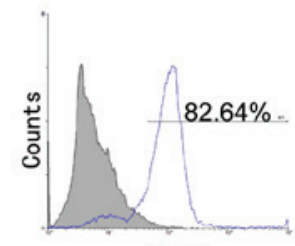

CD105

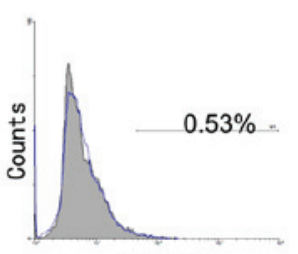

HLA-DR

Figure 2. Immunophenotyping by fluorescence-activated cell sorting of cells isolated from GC tissues and corresponding ovarian metastatic tissue. A heterogeneous, pooled population of cells at passage 4 was analyzed using flow cytometry to detect the expression of cell surface markers. The results show forward and side scatter, and in each plot, the X-axis shows relative fluorescence and the Y-axis shows the number of events. Histograms show the isotype control (gray peaks) vs. specific antibody staining (blue peaks) and the percentage of cells positive for the selected molecules. GC, gastric cancer; MSCs, mesenchymal stem cells; hGC-MSCs, human gastric cancer-MSCs; hGCOM-MSCs, human gastric cancer ovarian metastatic tissue-MSCs; CD, cluster of differentiation; HLA, human leukocyte antigen.

passage 4 contained the MSC population. These experiments were performed with the objective of isolating a population of pluripotent-like stem cells derived from human GC and its ovarian metastatic deposits. The present study revealed that the two types of cells were $\mathrm{CD}^{+} 3^{+}, \mathrm{CD} 90^{+}, \mathrm{CD}_{105^{+}}$,
CD34-, CD45- and HLA-DR-, which indicated characteristics of MSCs $(6,25,26)$. The hGC-MSCs at passage 4 expressed high levels of CD73 (78.58\%), CD90 (74.55\%) and CD105 $(77.15 \%)$. However, they were negative for the hematopoietic marker CD34 (0.74\%), CD45 (0.18\%) and HLA-DR (0.20\%). 


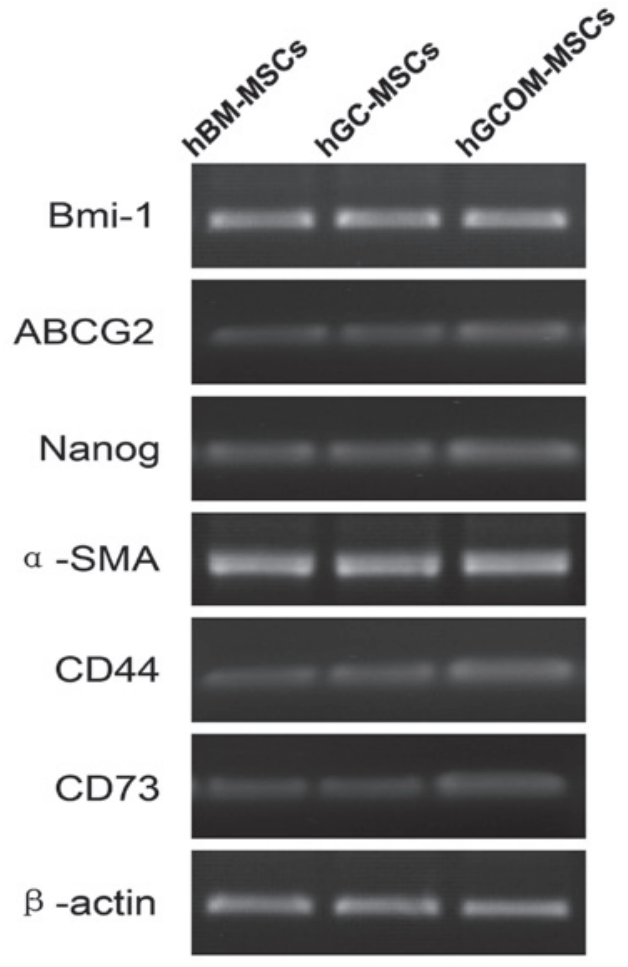

Figure 3. Gene expression of Bmi-1, ABCG2, Nanog, $\alpha$-SMA, CD44, CD73 and $\beta$-actin in hBM-MSCs, hGC-MSCs and hGCOM-MSCs. ABCG2, ATP-binding cassette sub-family G member 2; $\alpha$-SMA, $\alpha$-smooth muscle actin; $\mathrm{CD}$, cluster of differentiation; MSC, mesenchymal stem cells; hGC-MSCs, human gastric cancer-MSCs; hGCOM-MSCs, human gastric cancer ovarian metastatic tissue-MSCs; hBM-MSCs, human bone marrow-MSCs.

The hGCOM-MSCs at passage 4 also expressed high levels of CD73 (83.24\%), CD90 (76.32\%) and CD105 (82.64\%), but were negative for the hematopoietic markers CD34 (0.87\%), CD45 (0.66\%) and HLA-DR (0.53\%), indicating the mesenchymal lineage of these cells (Fig. 2). Taken together, these results demonstrated that cells isolated from human GC tissues and their ovarian metastatic tissues possess the characteristics of MSC with regards to the expression of markers. RT-PCR results demonstrated that stem cell-associated genes, including Snail, Bmi-1, ABCG2 and Nanog, and mesenchymal lineage-associated genes, including $\alpha$-SMA, CD73, CD44 and $\beta$-actin, were all expressed in hBM-MSCs, hGC-MSCs and hGCOM-MSCs (Fig. 3).

Animal studies. hGC-MSCs and hGCMO-MSCs failed to form tumors in NOD/SCID mice after $>2$ weeks (Fig. 4). However, GC cells (BGC-823) used as xenograft controls were able to form tumors 2 weeks after transplantation. Tumor formation was followed for 4 weeks.

Differentiation potential of $h G C-M S C s$ and hGCOM-MSCs. In addition to their colony-forming ability and the expression of specific antigens on their cell surfaces, the capacity for tri-lineage differentiation is a key property of MSCs (27). The differentiation potential was evaluated by culturing the cells in osteogenic media. Long-term cultures (3 weeks) of cells grown in the presence of osteogenic media demonstrated the capacity to form Alizarin Red-positive condensed nodules with high levels of calcium covering the entire wells. The deposits were sparsely scattered throughout the adherent layer as single mineralized zones (Fig. 5A).

In addition, the expression levels of ALP, OPN, OSC and DMP-1 genes, considered to be osteo-specific genes, were analyzed at the mRNA level after 3 weeks of osteoinduction. The expression of these mRNAs (relative to Actb) in differentiated hGC-MSCs and hGCOM-MSCs was higher than that in undifferentiated hGC-MSCs and hGCOM-MSCs (Fig. 5B). These results indicate that hGC-MSCs and hGCOM-MSCs cultured in osteogenic media can differentiate into osteoblast-like cells.

\section{Discussion}

MSCs are a subset of non-hematopoietic adult stem cells that originate from the mesoderm. They possess self-renewal ability and exhibit multilineage differentiation into not only mesoderm-lineages, including chondrocytes, osteocytes and adipocytes, but also ectodermal cells and endodermal cells (28). MSCs exist in almost all tissues. They can be easily isolated from the bone marrow, adipose tissue, umbilical cord, fetal liver, muscle and lung, and can be successfully expanded in vitro (29). Due to a lack of specific markers to define MSCs, their identification depends on their ability to adhere rapidly to tissue culture plastic, a panel of surface markers, including CD31, CD34, CD45, CD29, CD90 and CD105, as well as showing multilineage differentiation potential. The complexity of MSC interaction with the tumor microenvironment means that tumor growth and tumor metastasis can be affected by MSCs, directly or indirectly (30).

MSCs also exist in almost all tissues [needs citation]. Although it is hypothesized that bone marrow functions as a reservoir for disseminated tumor cells and that metastasis to secondary organs occurs due to the recirculation of disseminated tumor cells from bone marrow, the precise mechanisms remain to be elucidated. It was reported that in brain tumors affecting adult patients, tumor cells with stem-like characteristics have only been isolated from high-grade gliomas (31). By contrast, data from the present study demonstrated that tumor cells with stem cell-like can be isolated from GC and its ovarian metastatic tissues in the same patients (6/40). Further analysis of hGC-MSCs and hGCOM-MSCs cultures demonstrated that the isolated cells expressed a complex molecular profile that combined mesenchymal and gastric elements, suggesting a high potential of plasticity. Assessing in vivo tumorigenicity, it was verified that non self-renewing cells did not form tumors when engrafted into immunodeficient mice, whereas the three long-term self-renewing ones assessed generated tumors similar to the histological and molecular profile of the original human lesion.

Our success in derivation of self-renewing oncospheres from ovarian metastases of GC is comparable with or above the 50\% average success rate. Cao et al (24) initially reported the existence of MSC-like cells that can be isolated in vitro from GC tissues. In contrast to GC cells, these cells share most of the biochemical characteristics of hBM-MSCs, but also display unique characteristics, attributable to their unique locations. Our observation suggests that the ability to derive cells with properties akin to gastric progenitors or gastric stem cells from ovarian metastases of GC could be more dependent 


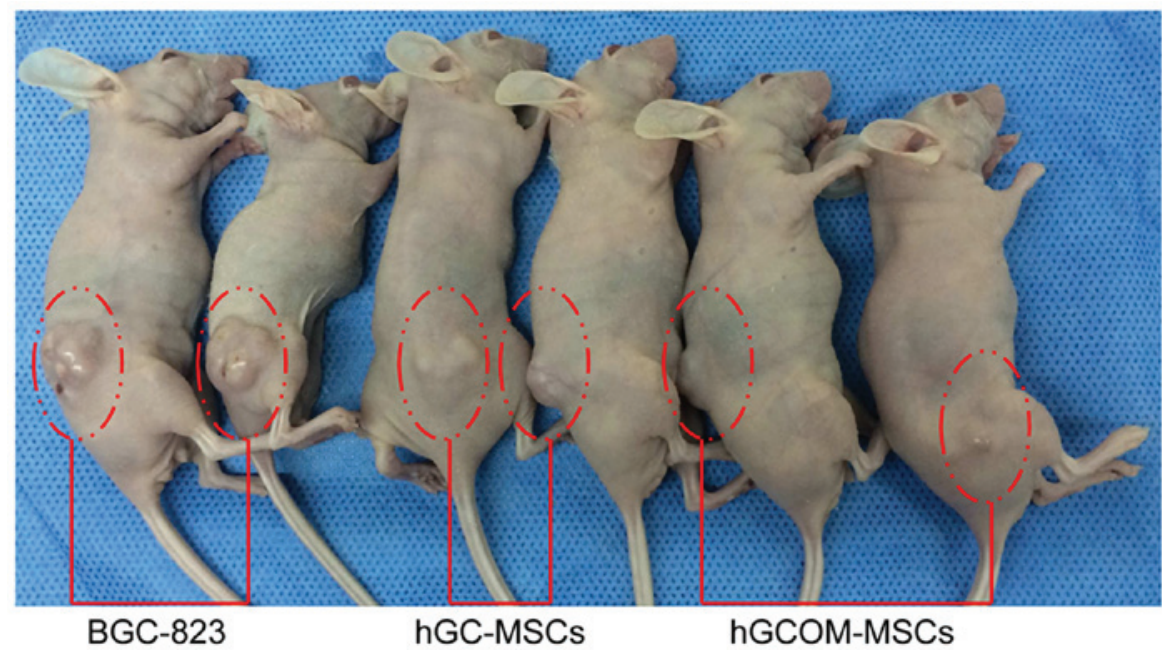

Figure 4. hGC-MSCs, hGCOM-MSCs and BGC-823 cells were injected into nude mice for 4 weeks. Tumor formation was observed in mice injected with BGC-823 cells but not hGC-MSCs or hGCOM-MSCs. MSCs, mesenchymal stem cells; hGC-MSCs, human gastric cancer-MSCs; hGCOM-MSCs, human gastric cancer ovarian metastatic tissue-MSCs.

A

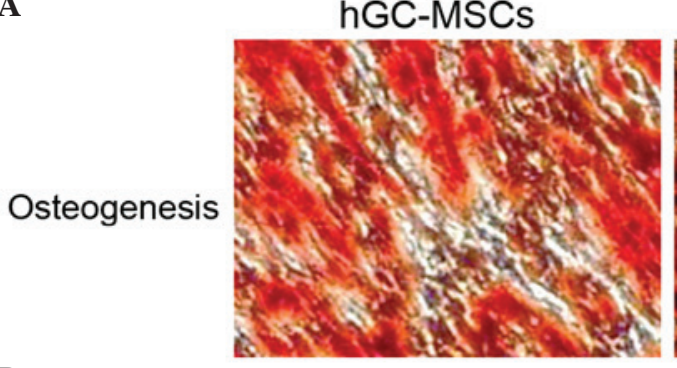

hGCOM-MSCS

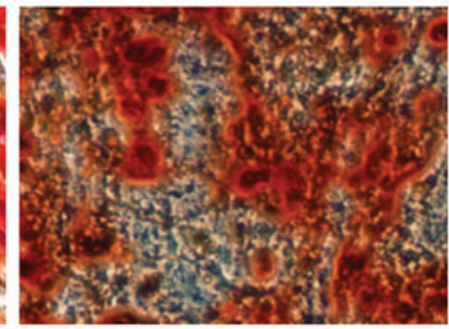

OPN

OSC
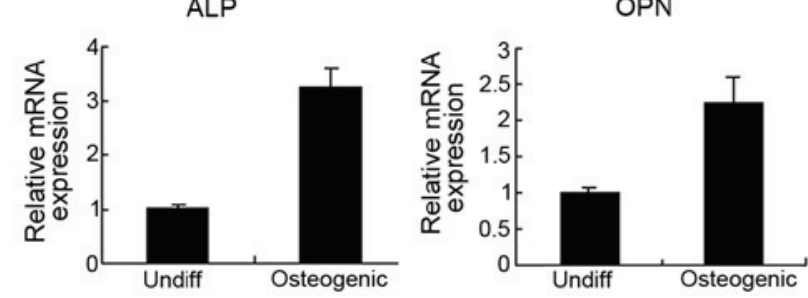

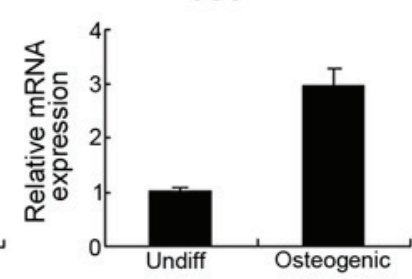

Negative

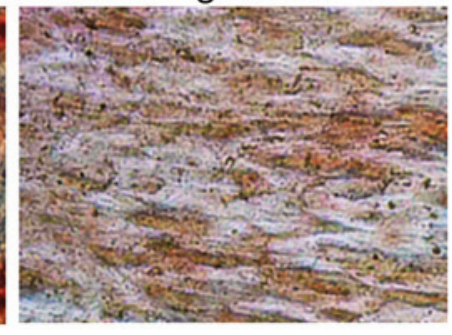

DMP-1

Figure 5. Osteogenic differentiation in hGC-MSCs and hGCOM-MSCs. (A) Mineralized deposit identified by Alizarin Red staining in cells grown in osteogenic medium for 3 weeks. Alizarin Red staining of hGC-MSCs and hGCOM-MSCs as viewed under the light microscope (magnification, x100). (B) Reverse transcription-polymerase chain reaction was used to determine the difference in gene expression profiles of osteoblastic-specific markers (ALP, OPN, OSC and DMP-1) between 1 month osteoblastic-differentiated cells and undifferentiated cells (Undiff). MSCs, mesenchymal stem cells; hGC-MSCs, human gastric cancer-MSCs; hGCOM-MSCs, human gastric cancer ovarian metastatic tissue-MSCs; ALP, alkaline phophatase; DMP-1, dentin matrix acidic phosphoprotein 1; OSC, osteocalcin; OPN, osteopontin.

on the absolute numbers of cells endowed with progenitor or stem properties within the cultured tumor sample, than on the optimization of culture conditions for each histological tumor sub-type. Further experiments are required to address this issue.

In order to further investigate the tumorigenicity of the isolated human gastric MSCs and ovarian metastatic MSCs, these cells were injected into nude mice and the results revealed that human gastric MSCs and ovarian metastatic MSCs were not able to form tumors even 1 month after initial injection, suggesting that human GC tissue MSCs and ovarian metastatic MSCs have not undergone transformation and are MSC-like cells but not CSCs. The long prevailing model of metastasis recognizes the importance of 'seed' and 'soil' for metastatic progression. An increasing amount of attention has focused on understanding the molecular and genetic factors that confer an intrinsic metastatic advantage to certain tumor cells. In addition, changes occurring within distant tissues, creating a 'soil' conducive for tumor invasion, have been largely neglected. Bone marrow-derived stem cells emerged as key players in initiating these early changes, creating a receptive microenvironment at designated sites for distant tumor growth and establishing the 'pre-metastatic niche' (32). This insight into the earliest stages in the metastatic cascade revises our concept of the metastatic 'microenvironment' to include physiological cells recruited from the bone marrow. Understanding the cellular and molecular cross-talk between 'seed' and 'soil' may improve our understanding of the factors that govern 
site-specific patterning in metastasis and the phenomenon of tumor dormancy. This may lead to therapeutic strategies to detect and prevent metastasis at its earliest inception. Human gastric MSCs and ovarian metastatic MSCs may be one of the most important components of the cancer 'niche.' On one hand, human GC MSCs may support the growth of tumor cells by cell-cell interaction. Alternatively, MSCs within human ovarian metastases of GC may contribute to the vasculature and extracellular matrix for the development of these tumor cells (33).

To the best of our knowledge, the present study is the first to demonstrate a distinct population of benign ovarian metastases of GC cells with stem cell-like properties. Further studies on the role of these cells in the initiation, development and/or progression of ovarian metastases from GC are warranted.

\section{Acknowledgements}

The present study was supported by the Hunan Province Health Department of China (grant no. B2013101, to Professor Chaohui Zuo) and Hunan Province Natural Science Foundation of China (grant no. 2015JJ6063), and the National Natural Science Foundation of China (grant no. 81500150, to Dr Man Xia).

\section{References}

1. Bou Kheir T, Futoma-Kazmierczak E, Jacobsen A, Krogh A, Bardram L, Hother C, Grønbæk K, Federspiel B, Lund AH and Friis-Hansen L: miR-449 inhibits cell proliferation and is down-regulated in gastric cancer. Mol Cancer 10: 29, 2011.

2. Fock KM and Ang TL: Epidemiology of Helicobacter pylori infection and gastric cancer in Asia. J Gastroenterol Hepatol 25: 479-486, 2010 .

3. Jemal A, Bray F, Center MM, Ferlay J, Ward E and Forman D: Global cancer statistics. CA Cancer J Clin 61: 69-90, 2011.

4. Leung WK, Wu MS, Kakugawa Y, Kim JJ, Yeoh KG, Goh KL, Wu KC, Wu DC, Sollano J, Kachintorn U, et al: Screening for gastric cancer in Asia: Current evidence and practice. Lancet Oncol 9: 279-287, 2008.

5. Cunningham D and Chua YJ: East meets west in the treatment of gastric cancer. N Engl J Med 357: 1863-1865, 2007.

6. Yilmaz M and Christofori G: Mechanisms of motility in metastasizing cells. Mol Cancer Res 8: 629-642, 2010.

7. Vignot $S$ and Soria JC: Discrepancies between primary tumor and metastasis: Impact on personalized medicine. Bull Cancer 100: 561-568, 2013 (In French).

8. Wang J, Shi YK, Wu LY, Wang JW, Yang S, Yang JL, Zhang HZ and Liu SM: Prognostic factors for ovarian metastases from primary gastric cancer. Int J Gynecol Cancer 18: 825-832, 2008.

9. Bianco P, Robey PG and Simmons PJ: Mesenchymal stem cells: Revisiting history, concepts and assays. Cell Stem Cell 2: 313-319, 2008.

10. Kato N, Hayasaka T, Takeda J, Osakabe M and Kurachi H: Ovarian tumors with functioning stroma: A clinicopathologic study with special reference to serum estrogen level, stromal morphology and aromatase expression. Int J Gynecol Pathol 32: 556-561, 2013.

11. Calloni R, Cordero EA, Henriques JA and Bonatto D: Reviewing and updating the major molecular markers for stem cells. Stem Cells Dev 22: 1455-1476, 2013.

12. Droujinine IA, Eckert MA and Zhao W: To grab the stroma by the horns: From biology to cancer therapy with mesenchymal stem cells. Oncotarget 4: 651-664, 2013.

13. Cuiffo BG and Karnoub AE: Mesenchymal stem cells in tumor development: Emerging roles and concepts. Cell Adh Migr 6: $220-230,2012$
14. Yang X, Hou J, Han Z, Wang Y, Hao C, Wei L and Shi Y: One cell, multiple roles: Contribution of mesenchymal stem cells to tumor development in tumor microenvironment. Cell Biosci 3: 5, 2013.

15. Xu MH, Gao X, Luo D, Zhou XD, Xiong W and Liu GX: EMT and acquisition of stem cell-like properties are involved in spontaneous formation of tumorigenic hybrids between lung cancer and bone marrow-derived mesenchymal stem cells. PLoS One 9: e87893, 2014

16. Ono M, Kosaka N, Tominaga N, Yoshioka Y, Takeshita F, Takahashi RU, Yoshida M, Tsuda H, Tamura K and Ochiya T: Exosomes from bone marrow mesenchymal stem cells contain a microRNA that promotes dormancy in metastatic breast cancer cells. Sci Signal 7: ra63, 2014.

17. Sung SY, Liao CH, Wu HP, Hsiao WC, Wu IH, Jinpu, Yu, Lin SH and Hsieh CL: Loss of let-7 microRNA upregulates IL-6 in bone marrow-derived mesenchymal stem cells triggering a reactive stromal response to prostate cancer. PLoS One 8: e71637, 2013.

18. Konno Y, Dong P, Xiong Y, Suzuki F, Lu J, Cai M, Watari H, Mitamura T, Hosaka M, Hanley SJ, et al: MicroRNA-101 targets EZH2, MCL-1 and FOS to suppress proliferation, invasion and stem cell-like phenotype of aggressive endometrial cancer cells Oncotarget 5: 6049-6062, 2014.

19. Liu Z, Jiang Z, Huang J, Huang S, Li Y, Sheng F, Yu S, Yu S and Liu X: Mesenchymal stem cells show little tropism for the resting and differentiated cancer stem cell-like glioma cells. Int J Oncol 44: 1223-1232, 2014.

20. Ochs K, Sahm F, Opitz CA, Lanz TV, Oezen I, Couraud PO, von Deimling A, Wick W and Platten M: Immature mesenchymal stem cell-like pericytes as mediators of immunosuppression in human malignant glioma. J Neuroimmunol 265: 106-116, 2013.

21. Bian ZY, Li G, Gan YK, Hao YQ, Xu WT and Tang TT: Increased number of mesenchymal stem cell-like cells in peripheral blood of patients with bone sarcomas. Arch Med Res 40: 163-168, 2009.

22. Kirkland SC: Type I collagen inhibits differentiation and promotes a stem cell-like phenotype in human colorectal carcinoma cells. Br J Cancer 101: 320-326, 2009.

23. Marrelli M, Paduano F and Tatullo M: Cells isolated from human periapical cysts express mesenchymal stem cell-like properties. Int J Biol Sci 9: 1070-1078, 2013.

24. Cao H, Xu W, Qian H, Zhu W, Yan Y, Zhou H, Zhang X, Xu X, Li J, Chen Z and Xu X: Mesenchymal stem cell-like cells derived from human gastric cancer tissues. Cancer Lett 274: 61-71, 2009.

25. Mamidi MK, Pal R, Govindasamy V, Zakaria Z and Bhonde R: Treat the graft to improve the regenerative ability of the host. Med Hypotheses 76: 599-601, 2011.

26. Xu J, Wang W, Kapila Y, Lotz J and Kapila S: Multiple differentiation capacity of STRO-1 $1^{+}$CD $146^{+}$PDL mesenchymal progenitor cells. Stem Cells Dev 18: 487-496, 2009.

27. Ribitsch I, Burk J, Delling U, Geißler C, Gittel C, Jülke H and Brehm W: Basic science and clinical application of stem cells in veterinary medicine. Adv Biochem Eng Biotechnol 123: 219-263, 2010.

28. Dezawa M, Ishikawa H, Itokazu Y, Yoshihara T, Hoshino M, Takeda S, Ide C and Nabeshima Y: Bone marrow stromal cells generate muscle cells and repair muscle degeneration. Science 309: 314-317, 2005.

29. Barberini DJ, Freitas NP, Magnoni MS, Maia L, Listoni AJ, Heckler MC, Sudano MJ, Golim MA, da Cruz LandimAlvarenga $\mathrm{F}$ and Amorim RM: Equine mesenchymal stem cells from bone marrow, adipose tissue and umbilical cord: Immunophenotypic characterization and differentiation potential. Stem Cell Res Ther 5: 25, 2014.

30. Cuiffo BG and Karnoub AE: Mesenchymal stem cells in tumor development: Emerging roles and concepts. Cell Adh Migr 6: 220-230, 2012.

31. Thirant C, Bessette B, Varlet P, Puget S, Cadusseau J, Tavares Sdos R, Studler JM, Silvestre DC, Susini A, Villa C, et al: Clinical relevance of tumor cells with stem-like properties in pediatric brain tumors. PLoS One 6: e16375, 2011.

32. Kaplan RN, Riba RD, Zacharoulis S, Bramley AH, Vincent L, Costa C, MacDonald DD, Jin DK, Shido K, Kerns SA, et al: VEGFR1-positive haematopoietic bone marrow progenitors initiate the pre-metastatic niche. Nature 438: 820-827, 2005.

33. Lescarbeau RM, Seib FP, Prewitz M, Werner C and Kaplan DL: In vitro model of metastasis to bone marrow mediates prostate cancer castration resistant growth through paracrine and extracellular matrix factors. PLoS One 7: e40372, 2012. 